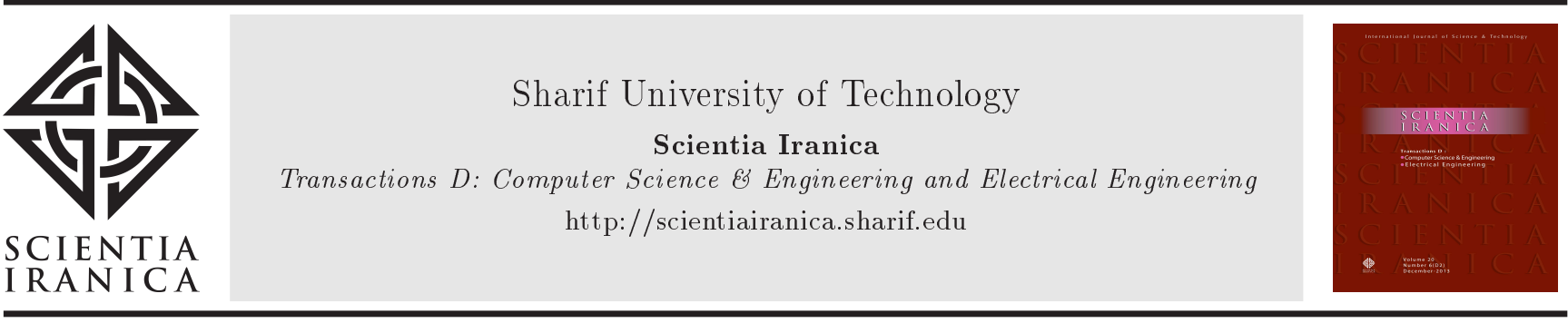

\title{
The effects of demand response on security-constrained unit commitment
}

\author{
E. Zarei ${ }^{a, *}$, M.H. Hemmatpour ${ }^{\mathrm{b}}$, and M. Mohammadian ${ }^{\mathrm{a}}$ \\ a. Department of Electrical Engineering, Faculty of Engineering, Shahid Bahonar University of Kerman, Kerman, Iran. \\ b. Department of Electrical Engineering, Faculty of Engineering, Jahrom University, Jahrom, Fars, Iran.
}

Received 9 January 2016; received in revised form 18 August 2017; accepted 14 October 2017

\author{
KEYWORDS \\ Bender's \\ decomposition; \\ Demand response; \\ Real-time pricing; \\ Restructured power \\ market; \\ SCUC.
}

\begin{abstract}
This paper aims to study the effect of the hourly Demand Response (DR) on Security-Constrained Unit Commitment (SCUC) problem considering economic and security objectives. The demand side participation can solve some of the electricity market problems. In this paper, the DR is taken into consideration as one of the Demand Side Management (DSM) parts. The DR consists of fixed and responsive loads. The fixed loads can be satisfied under any circumstances, and responsive loads can reduce or shift to other operation hours. A combination of SCUC with DR is a complex and mixed integer non-linear problem. The bender's decomposition is used as an optimization technique for solving this problem. This technique solves the problem by decomposing it into master and sub problems. One of the advantages of this technique is short processing time. The performance and effectiveness of the proposed method are evaluated with respect to 6-, $24-$, and 118-bus test systems.

(C) 2019 Sharif University of Technology. All rights reserved.
\end{abstract}

\section{Introduction}

The restructured power market is composed of several independent players who benefit from the DR. These players are GENCOs, TRANSCOs, DISCOs, ISO, and other different parts [1]. The Independent System Operator (ISO) should schedule available resources for satisfying the demand by considering security at the minimum cost. The GENCOs submit their bids to the ISO for satisfying next-day demands [1,2]. In the electricity market, the marginal price is defined based on the last scheduled generator [3]. In such a market, the elasticity of demand is often thought to range from zero in the real-time markets, and the

\footnotetext{
*. Corresponding author. Tel./Fax: +983431325900 E-mail addresses: Zareii.ebrahim@gmail.com (E. Zarei); m.h.hematpour@jahrom.ac.ir (M.H. Hemmatpour); M.Mohammadian@uk.ac.ir (M. Mohammadian)
}

doi: $10.24200 /$ sci. 2017.4536 demand side has no participation in the electricity market [4]. Thus, the generators submit their bids at a price much higher than the marginal market price [5]. The spike in prices also occurs at peak hours when sufficient reserve is not available. To compensate for this absence of power, the generators with high marginal cost are employed [6]. In such a cycle, the DR participation is a good, economical method to overcome the system constraint and spike in prices [7]. These problems occur, when the hourly power dispatch does not consider the DR role in the market. Incorporating the DR in the market structure is profitable for all of the market participants $[8,9]$. The demand side management consists of the distributed generation, the energy storage site, and the DR [10]. Here, it is focused on the DR considering a combination of fixed and responsive loads. The DR is an opportunity for consumers to save money through reductions in the peak demand. The DR has the ability to defer the construction of new power plants and power delivery systems such as those reserved for use during peak 


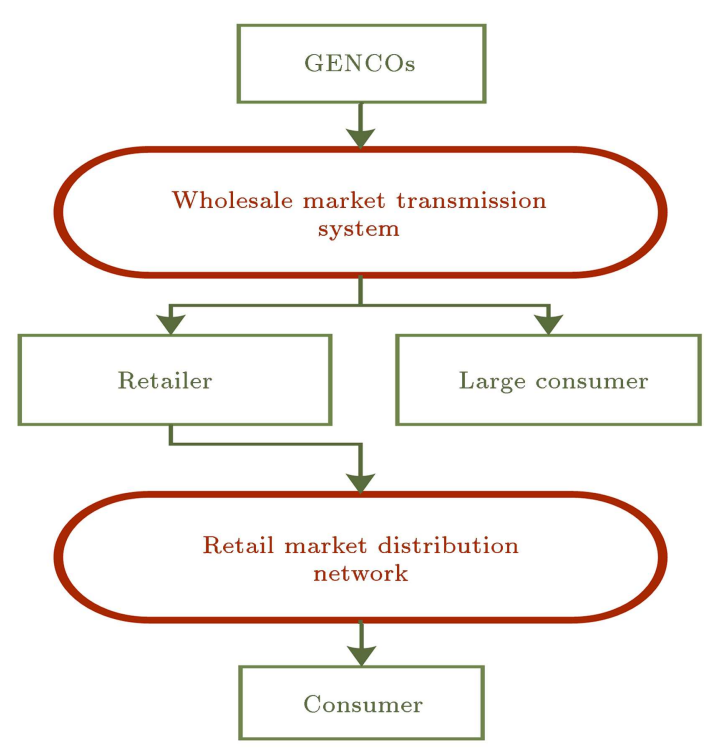

Figure 1. Energy market structure.

times. The DR can be categorized into two subgroups: economical and emergency DRs.

In addition, the emergency one curtails loads of the network according to the network's condition, and the economical one is curtailed voluntarily based on market prices. In a restructured power market, electricity prices will fluctuate with time and place of loads [11-13]. The structure of the energy market is shown in Figure 1. The GENCOs and TRANSCOs submit their information to the ISO and DISCOs aggregate loads data for the ISO. Achieving a dynamic market with the best operation schedule is the main objective that ISO tries to realize by incorporating the DR in SCUC. This will make a new opportunity for customers and market participants [14].

In [15], to maximize social welfare, a new market clearing process was supposed in which customers submit their bids for buying energy. In this model, a power market with the auction pool is introduced; accordingly, the buyer or seller submits their bids to the ISO for making the right decision. The ISO gathers all submitted bids and solves the unit commitment problem to obtain the minimum cost. The most important objective of the ISO is to maximize the social welfare, which is defined as a summation of consumption and production surplus. In [16], a multidirectional auction was introduced; accordingly, the market participants attempt to find an equilibrium point for submitting their bids. Here, the main difference is that the seller and the market operators attempt to maximize the benefit according to their needs. In [17], the load constraints with the hourly biding mechanism were modeled on the day ahead of the auction. For this purpose, security was considered as an ancillary service, and the price was the signal that specified the unit status. In [18], an iterative process was used to calculate the market price and the DR change. According to the last load profile, the amount and method of the DR combination were estimated. The authors in [19] discussed a new concept of the DR as a DR exchange (DRX) that could make a good trade-off between a buyer and a seller. In [20], the DR was incorporated into SCUC, and the objective function maximizes the social welfare with the bender's decomposition technique. As observed, the market clearing process was conducted by either direct or iterative approaches. In the direct approach, the elastic characteristic of the demand price is used to set the demand.

The operation and transmission constraints are not considered in this approach. In addition, the iterative process is a time-consuming process, and the feasible solution may not be found by this method. Herein, the operation constraints of the DR and transmission line are not considered.

In this paper, the SCUC problem along with the DR is solved by bender's decomposition technique. This technique converges to the result at a proper time.

\section{The market clearing model}

The GENCOs and the loads submit their simple bids (it could also be complex) to the ISO. The transmission constraints and probabilities are considered in the base case. The objective function is aimed at maximizing the social welfare, which is defined by the profit obtained from selling the electricity power mines and reducing the generation cost. The hourly SCUC for the complex bids specifies the unit status and power dispatch for each unit and the hourly DR according to the submitted bids $[1,11]$.

\section{Load offer}

The DR consists of fixed and responsive loads. The fixed loads are associated with price takers and should be fully satisfied in the market clearing process. The fixed load prices reduce as demand increases.

The responsive loads could reduce or shift to other operation hours. This type of loads should offer the amount of hourly load and their price to the ISO. The load characteristic with their constraints is imposed on the SCUC problem. These constraints consist of the minimum on/off time, up/down rate, bus load, minimum hourly curtailment, and maximum load curtailment [15-17].

\section{Formulation of SCUC with the DR and bender's decomposition}

This is a mixed integer non-linear optimization program. The bender's decomposition technique is used 


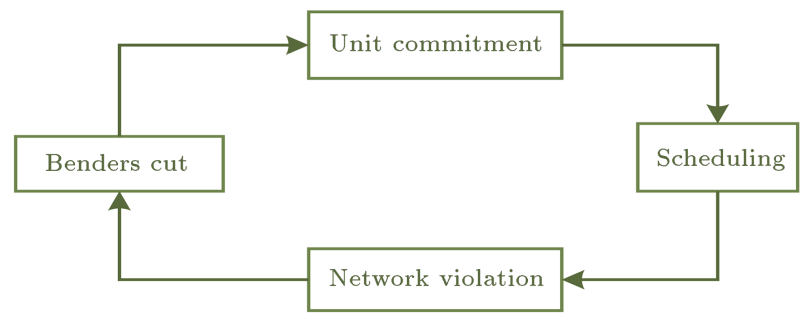

Figure 2. SCUC with bender's decomposition.

in this formulation to reduce a large amount of computation and time-consuming process. By using this technique, the problem is divided into master and sub problems. The master problem specifies the main unit commitment and units and loads status with their schedule. Checking the feasibility and probability condition is done in sub problems. If any violation occurs, the bender's decomposition cuts the process and adds to the master problem according to the violation. In the next iteration, the master problem is solved by this additional constraint. This iterative process between the master and sub problems continues until all constraints are satisfied. These iterative processes for the SCUC problem and DR are depicted in Figure $2[1,2]$.

\section{Formulation}

The decomposition technique is applied to solve the mixed integer non-linear program. Therefore, the master and sub problems along with the related constraints are briefly discussed below.

\subsection{Master problem}

The objective function of the master problem is to schedule the generation unit and loads by the prevailing system constraint in the base case to maximize the social welfare. The objective function of the master problem is shown in Eq. (1):

$$
\begin{aligned}
\operatorname{Max} & \left\{\sum_{t=1}^{N T} \sum_{b=1}^{N B} C B_{b t}\left(D_{b t}\right)-\sum_{t=1}^{N T} \sum_{i=1}^{N G}\left[F_{c i}\left(P_{i t}\right) * I_{i t}\right.\right. \\
& \left.\left.+S U_{i t}+S D_{i t}\right]\right\} .
\end{aligned}
$$

\subsection{System constraints}

1. The power balance: Eq. (2) demonstrates the power balance in the power system. By this constraint, the power generation should be equal to the demand plus the network losses.

$$
\sum_{i=1}^{N G} P_{i t} * I_{i t}=\sum_{b=1}^{N B} D_{b t}+P_{L, t}, \quad t=1,2, \ldots, N T .
$$

2. The system spinning and operating reserve: To maintain system reliability, the adequate spinning and operating reserves are required, which are defined as follows:

$$
\begin{aligned}
& \sum_{i=1}^{N G} R_{S, i t} * I_{i t} \geq R_{S, t}, \quad t=1,2, \ldots, N T \\
& \sum_{i=1}^{N G} R_{o, i t} * I_{i t} \geq R_{o, t}, \quad t=1,2, \ldots, N T .
\end{aligned}
$$

3. The ramp rate: for each unit, the output is limited by the up/down ramp rate at each hour as follows:

$$
\begin{aligned}
& P_{i t}-P_{i(t-1)} \leq\left[1-I_{i t}\left(1-I_{i(t-1)}\right)\right] U R_{i} \\
& \quad+I_{i t}\left(1-I_{i(t-1)}\right) P_{i, \min }, \\
& i=1,2, \ldots, N G ; \quad t=1,2, \ldots, N T \\
& P_{i(t-1)}-P_{i t} \leq\left[1-I_{i(t-1)}\left(1-I_{i t}\right)\right] D R_{i} \\
& \quad+I_{i(t-1)}\left(1-I_{i t}\right) P_{i, m i n}, \\
& i=1,2, \ldots, N G ; \quad t=1,2, \ldots, N T .
\end{aligned}
$$

4. The minimum on/off time: thermal units cannot change their status suddenly because each unit has a cool and warm up time. These limitations are explained in the following equations:

$$
\begin{aligned}
& {\left[X_{i(t-1)}^{o n}-T_{i}^{o n}\right]\left[I_{i(t-1)}-I_{i t}\right] \geq 0,} \\
& i=1,2, \ldots, N G ; \quad t=1,2, \ldots, N T \\
& {\left[X_{i(t-1)}^{o f f}-T_{i}^{o f f}\right]\left[I_{i t}-I_{i(t-1)}\right] \geq 0,} \\
& i=1,2, \ldots, N G ; \quad t=1,2, \ldots, N T .
\end{aligned}
$$

5. Power output constraints: The power output of each unit has its lower and upper bounds as follows:

$$
\begin{aligned}
& P_{i, \min } * I_{i t} \leq P_{i t} \leq P_{i, \max } * I_{i t} \\
& i=1,2, \ldots, N G ; \quad t=1,2, \ldots, N T .
\end{aligned}
$$

This constraint is imposed to keep the power generation of each unit inside this band $[1,21,22]$.

\subsection{Load constraints}

The following equations show the load constraints in 
the market. Eq. (10) shows that the summation of fixed and responsive loads should be equal to the total loads at each bus. The minimum load curtailment and nonnegative responsive load are explained in Eqs. (10) and (11), respectively. The submitted responsive load is shown in Eq. (13). If the load is not curtailed, the responsive load schedule is submitted. The load of each bus should set their decrement and increment levels according to their ramp up/down limit rate, as given in Eqs. (14) and (15).

Loads such as generation units have the minimum on/off time of the loads, as explained in Eqs. (16) and (17). Loads use these constraints for restricting on/off status of the loads. These constraints demonstrate the minimum number of the hours that the loads can be curtailed or restored. Constraint (18) restricts the total daily load curtailment. The hourly load curtailment is the difference between the submitted responsive load and the scheduled responsive load. This term is positive when the load is curtailed, negative when the load is shifted to that hour, and zero when there is no load curtailment or shifting at that hour. By using this constraint, responsive loads may be shifted or curtailed $[14,20]$.

$$
\begin{aligned}
& D_{b t}=\mathrm{D}_{b t}^{f}+\mathrm{D}_{b t}^{r}, \quad b=1,2, \ldots, N B ; \quad t=1,2, \ldots, N T \\
& {\left[D_{b t}^{r, m a x}-D X_{b t}^{m i n}-D_{b t}^{r}\right] v_{b t} \geq 0} \\
& \quad b=1,2, \ldots, N B ; \quad t=1,2, \ldots, N T \\
& D_{b t}^{r} v_{b t} \geq 0 \quad b=1,2, \ldots, N B ; \quad t=1,2, \ldots, N T
\end{aligned}
$$$$
\left[D_{b t}^{r}-D_{b t}^{r, \max }\right]\left[1-v_{b t}\right] \geq 0
$$$$
b=1,2, \ldots, N B ; \quad t=1,2, \ldots, N T
$$$$
D_{b t}^{r}-D_{b(t-1)}^{r} \leq U R_{b},
$$$$
b=1,2, \ldots, N B ; \quad t=1,2, \ldots, N T
$$$$
D_{b(t-1)}^{r}-D_{b t}^{r} \leq D R_{b}
$$$$
b=1,2, \ldots, N B ; \quad t=1,2, \ldots, N T,
$$$$
\left[X_{b(t-1)}^{o n}-U T_{b}\right]\left[v_{b(t-1)}-v_{b t}\right] \geq 0,
$$$$
b=1,2, \ldots, N B ; \quad t=1,2, \ldots, N T,
$$$$
\left[X_{b(t-1)}^{o f f}-D T_{b}\right]\left[v_{b t}-v_{b(t-1)}\right] \geq 0
$$$$
b=1,2, \ldots, N B ; \quad t=1,2, \ldots, N T
$$

$$
\begin{gathered}
\sum_{t=1}^{N T}\left(D_{b t}^{r, \max }-D_{b t}^{r}\right) \leq E X_{b}^{\max }, \\
b=1,2, \ldots, N B .
\end{gathered}
$$

5.4. Sub problem and bender's decomposition The bender's decomposition technique is applied to solve this mixed integer non-linear optimization problem. The unit schedules are cleared by solving SCUC in the master problem. Then, the feasibility and contingencies of results are checked in sub problems. In the case of violation, hourly cuts (Relations (19) and (20)) add to the master problem in the next iteration. In sub problems, the power mismatch is minimized at all buses $[1,2,23]$.

$$
\begin{aligned}
\hat{w}_{t} & +\sum_{i=1}^{N G} \pi_{i t}\left(P_{i t} I_{i t}-\hat{P}_{i t} \hat{I}_{i t}\right) \\
& +\sum_{b=1}^{N B} \mu_{b t}\left(D_{b t}-\hat{D}_{b t}\right) \leq 0, \\
t & =1,2, \ldots, N T, \\
\hat{w}_{t}+ & \sum_{i=1}^{N G}\left(\bar{\pi}_{i t}-\bar{\pi}_{i t}\right)\left(P_{i t}-\hat{P}_{i t}\right) \\
& +\sum_{b=1}^{N B} \mu_{b t}\left(D_{b t}-\hat{D}_{b t}\right) \leq 0, \\
t & =1,2, \ldots, N T,
\end{aligned}
$$

where $\hat{w}_{t}$ shows the power mismatch in the base case for the current bus. In addition, $\pi_{i t}, \mu_{b t}, \bar{\pi}_{i t}$, and $\underline{\pi}_{i t}$ are the dual variables of hourly dispatch, the load balance equations, and the hourly generation constraint, respectively.

\section{Case studies}

The proposed methodology is implemented on the modified standard IEEE 6-bus, 24-bus, and 118-bus systems. The simulation is carried out by GAMS 23.3 software with a personal computer [23]. In these cases, ten percent of the total load is considered as responsive, while the rest is fixed. The consumption bid for the responsive loads is 15 \$.MW.

The operation constraints are:

1. The consumption bid is $15 \$ / \mathrm{MWh}$ for the responsive loads;

2. The minimum up-and-down time is $4 \mathrm{~h}$;

3. The minimum hourly and maximum daily load curtailment rates are considered as $5 \mathrm{MW}$ and $150 \mathrm{MW}$. 


\subsection{Case study 1: Six-bus system}

\subsubsection{Scenario 1: The base case}

For this base case, the SCUC problem is solved without considering DR. The operation cost and the processing time are obtained $\$ 137491.924$ and 3 min, respectively. The load is satisfied directly from three units at different hours. The hours that each unit generates power are shown in Figure 3. The hourly power generation over a period of one day is shown in Table 1. This table is summarized in Figure 4.

\subsubsection{Scenario 2: A 6-bus system with the DR in all buses}

By incorporating the DR into the SCUC, the total operation cost and the processing time are obtained

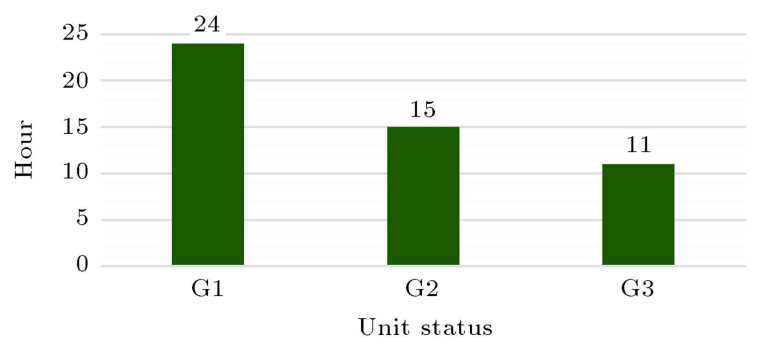

Figure 3. The unit status of the 6-bus system in the base case.

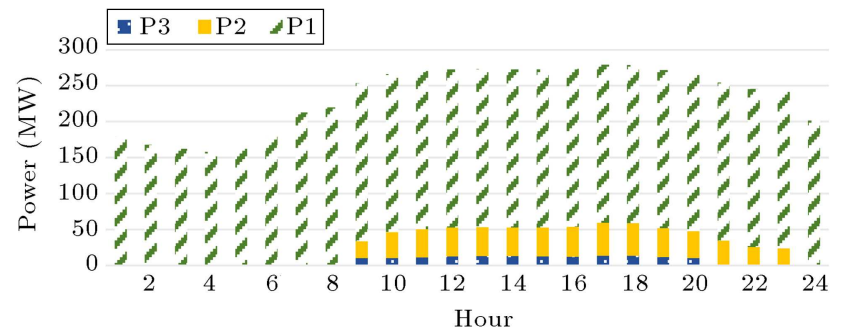

Figure 4. The aggregated power for the 6-bus system in the base case. as $\$ 124421.209$ and $5 \mathrm{~min}$, respectively. The economic point based on the DR saves $\$ 13070.715$ in comparison to the base case. The curtailed loads are depicted in Figure 5. The load curtailment is shown as a function of time deviation for each curtailed load in Figure 6.

The power generation, the unit status, and the aggregated power generation of each generator are shown in Table 2 and Figures 7 and 8, respectively.

The reasons for a decrease in the total operation cost in this scenario are summarized as follows:

1. Generation unit 2 almost generates fixed power far away from the marginal cost;

2. It is clear that the DR is the main reason to compensate the lack of power at the peak load hours. Therefore, the DR can reduce the total operation cost.

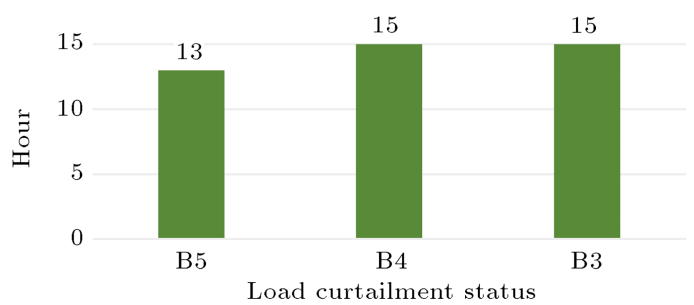

Figure 5. The load curtailment in the 6-bus system.

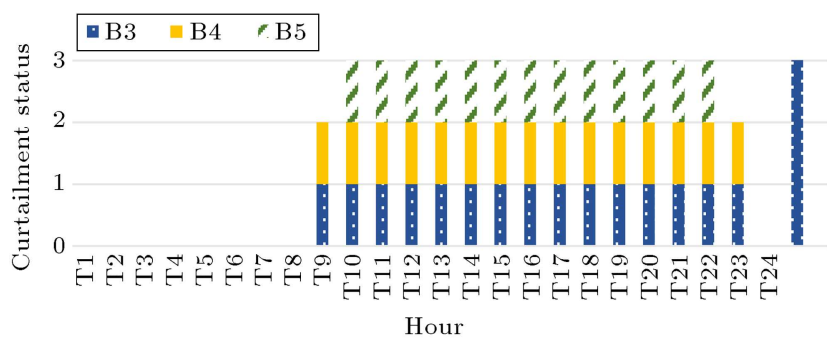

Figure 6. The total load curtailment in the 6-bus system.

Table 1. The power generation for the 6-bus system in the base case.

\begin{tabular}{lcccccccc}
\hline & \multicolumn{7}{c}{ Hour } \\
\cline { 2 - 9 } P1 & T1 & T2 & T3 & T4 & T5 & T6 & T7 & T8 \\
\cline { 2 - 9 } P2 & 179.2 & 168 & 162.4 & 156.8 & 162.4 & 179.2 & 212.8 & 220 \\
P3 & 0 & 0 & 0 & 0 & 0 & 0 & 0 & 0 \\
\cline { 2 - 9 } & T9 & T10 & T11 & T12 & T13 & T14 & T15 & T16 \\
\cline { 2 - 9 } P1 & 220 & 220 & 220 & 220 & 220 & 220 & 220 & 220 \\
P2 & 23.6 & 36 & 39.2 & 40 & 40.2 & 40 & 40 & 41.6 \\
P3 & 10 & 10 & 11.3 & 12.63 & 12.85 & 12.79 & 12.56 & 12.23 \\
\cline { 2 - 9 } & T17 & T18 & T19 & T20 & T21 & $\mathbf{T 2 2}$ & $\mathbf{T 2 3}$ & $\mathbf{T 2 4}$ \\
\cline { 2 - 9 } P1 & 220 & 220 & 220 & 220 & 220 & 220 & 220 & 201.6 \\
P2 & 45.8 & 45.8 & 40.4 & 37.6 & 25.6 & 25.4 & 23.6 & 0 \\
P3 & 13.6 & 13.18 & 11.42 & 10 & 0 & 0 & 0 & 0 \\
\hline
\end{tabular}


Table 2. The power generation for the 6-bus system with the DR.

\begin{tabular}{lcccccccc}
\hline & \multicolumn{7}{c}{ Hour } \\
\cline { 2 - 9 } P1 & T1 & T2 & T3 & T4 & T5 & T6 & T7 & T8 \\
\cline { 2 - 9 } P2 & 0 & 0 & 0 & 0 & 0 & 0 & 0 & 0 \\
P3 & 0 & 0 & 0 & 0 & 0 & 0 & 0 & 0 \\
\cline { 2 - 9 } & $\mathbf{T 9}$ & $\mathbf{T 1 0}$ & $\mathbf{T 1 1}$ & $\mathbf{T 1 2}$ & $\mathbf{T 1 3}$ & $\mathbf{T 1 4}$ & $\mathbf{T 1 5}$ & $\mathbf{T 1 6}$ \\
\cline { 2 - 9 } P1 & 220 & 220 & 220 & 220 & 220 & 220 & 220 & 220 \\
P2 & 0 & 0 & 10 & 12 & 10 & 12 & 12 & 10 \\
P3 & 12.528 & 19.4 & 19.48 & 20 & 19.48 & 20 & 19.48 & 20 \\
\cline { 2 - 9 } & $\mathbf{T 1 7}$ & $\mathbf{T 1 8}$ & $\mathbf{T 1 9}$ & $\mathbf{T 2 0}$ & $\mathbf{T 2 1}$ & $\mathbf{T 2 2}$ & $\mathbf{T 2 3}$ & $\mathbf{T 2 4}$ \\
\cline { 2 - 9 } P1 & 220 & 220 & 220 & 220 & 220 & 220 & 220 & 220 \\
P2 & 10 & 10 & 0 & 0 & 0 & 0 & 0 & 0 \\
P3 & 20 & 20 & 14.44 & 14.36 & 12.528 & 12.528 & 12.36 & 0 \\
\hline
\end{tabular}

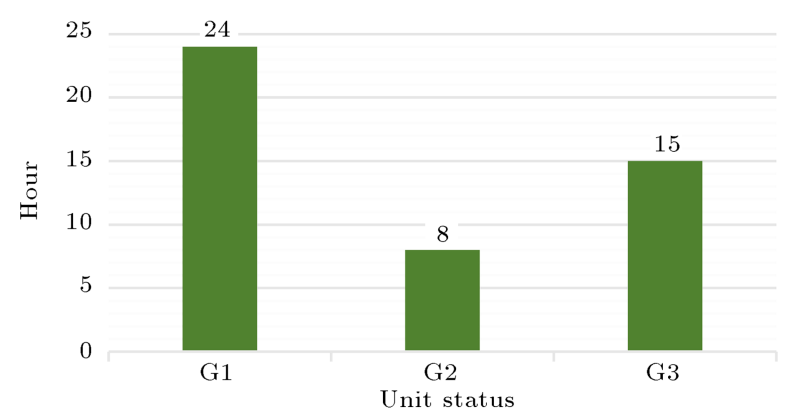

Figure 7. The unit status for the 6-bus with the DR.

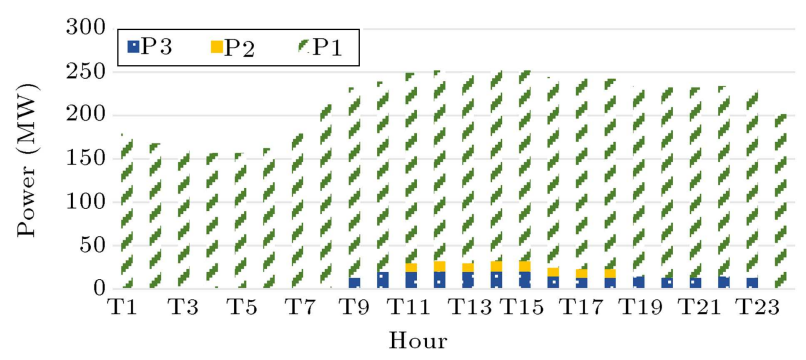

Figure 8. The aggregated power for the 6-bus with the DR.

The demands in SCUC problem with the DR (in the base case) and in a combination of SCUC problem with the DR are defined by D1 and D2 in Table 3, respectively. D1 is equal to D2 in the hours between T1 to T9. However, D2 is less than D1 in the other hour. The effect of the DR on the actual system load is shown in Figure 9. The DR shave the peak load that reduces the total operation cost, the spinning reserve, the transmission congestion, and the emission.

\subsection{Case study 2: 24-bus system \\ 6.2.1. Scenario 1: The SCUC problem in the base case}

This network has 33 generation units and 34 lines. The peak load of this network is $2821.5 \mathrm{MW}$. The SCUC

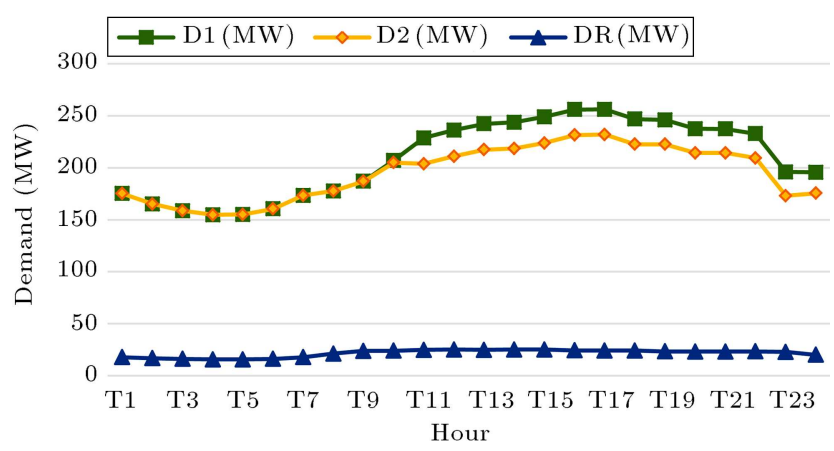

Figure 9. The DR effects on the demand curve for the 6-bus system.

problem is solved without considering the DR. The operation cost and the processing time are obtained as $\$ 373869.23$ and $18 \mathrm{~min}$, respectively. The unit status is shown in Figure 10. Based on the figure, 32 units participate in power generation, unit 15 is off, and almost most of them produce power throughout the whole day. Figure 10 shows the hours that each unit generates power.

\subsubsection{Scenario 2: A 24-bus system with the DR}

By incorporating the DR into the SCUC, the total operation cost and the processing time are obtained as $\$ 349897.73$ and $24 \mathrm{~min}$, respectively, saving the amount of $\$ 23971.5$ in comparison to the amount in the base case. Figure 11 shows the status of each load participation in the load curtailment. Herein, the buses with zero status, unlike one status, have no role in the load curtailment program. The status of each unit is shown in Figure 12.

In Table 4, the demands before combination with SCUC (D1) and after combination (D2) are clearly shown. The DR shaves the demand peak curve, and generators decrease their production levels. The effect of this demand shaving is shown in Figure 13. 
Table 3. The DR effects on the demand reduction for the 6-bus system.

\begin{tabular}{lcccccccc}
\hline & \multicolumn{7}{c}{ Hour } \\
\cline { 2 - 9 } D1 (MW) & $\mathbf{T 1}$ & $\mathbf{T 2}$ & $\mathbf{T 3}$ & $\mathbf{T 4}$ & $\mathbf{T 5}$ & $\mathbf{T 6}$ & $\mathbf{T 7}$ & T8 \\
\cline { 2 - 9 } D2 (MW) & 175.19 & 165.15 & 158.67 & 154.73 & 155.06 & 160.48 & 173.39 & 177.6 \\
DR (MW) & 17.92 & 16.8 & 16.24 & 15.68 & 15.68 & 16.24 & 17.92 & 21.28 \\
\cline { 2 - 9 } & $\mathbf{T 9}$ & $\mathbf{T 1 0}$ & $\mathbf{T 1 1}$ & $\mathbf{T 1 2}$ & $\mathbf{T 1 3}$ & $\mathbf{T 1 4}$ & $\mathbf{T 1 5}$ & $\mathbf{T 1 6}$ \\
\cline { 2 - 9 } D1 (MW) & 186.81 & 206.96 & 228.61 & 236.1 & 242.18 & 243.6 & 248.86 & 255.79 \\
D2 (MW) & 186.81 & 204.86 & 203.662 & 210.9 & 217.232 & 218.4 & 223.66 & 231.346 \\
DR (MW) & 23.86 & 23.997 & 24.948 & 25.2 & 24.948 & 25.2 & 25.2 & 24.443 \\
\cline { 2 - 9 } & $\mathbf{T 1 7}$ & $\mathbf{T 1 8}$ & $\mathbf{T 1 9}$ & $\mathbf{T 2 0}$ & $\mathbf{T 2 1}$ & $\mathbf{T 2 2}$ & $\mathbf{T 2 3}$ & $\mathbf{T 2 4}$ \\
\cline { 2 - 9 } D1 (MW) & 256 & 246.74 & 245.97 & 237.35 & 237.31 & 232.67 & 195.91 & 195.6 \\
D2 (MW) & 231.808 & 222.548 & 222.534 & 214.166 & 214.126 & 209.236 & 172.93 & 175.44 \\
DR (MW) & 24.192 & 24.192 & 23.436 & 23.184 & 23.184 & 23.436 & 23 & 20.16 \\
\hline
\end{tabular}

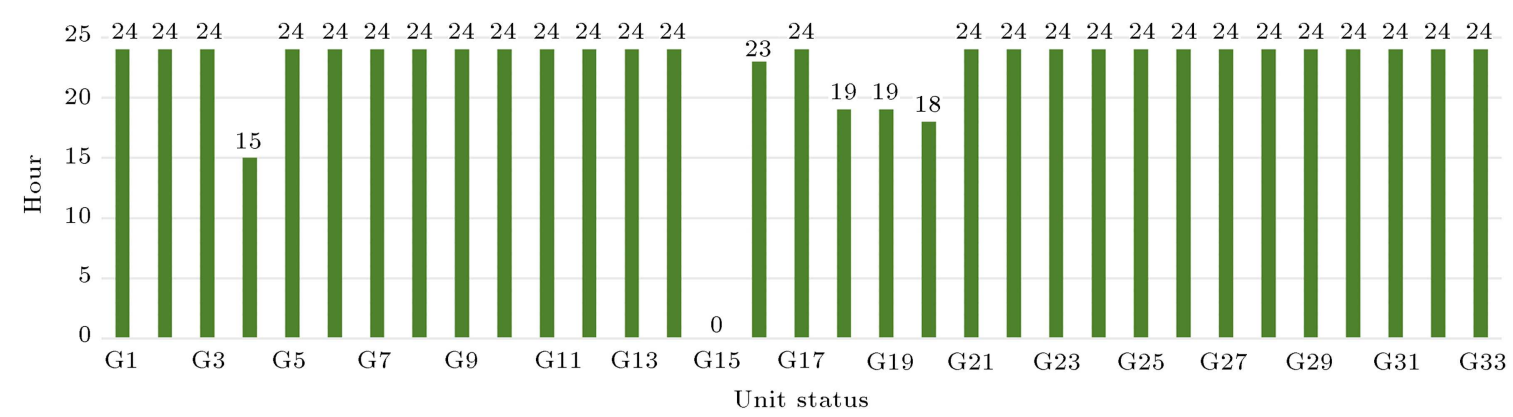

Figure 10. The unit status for the 24-bus system in the base case.

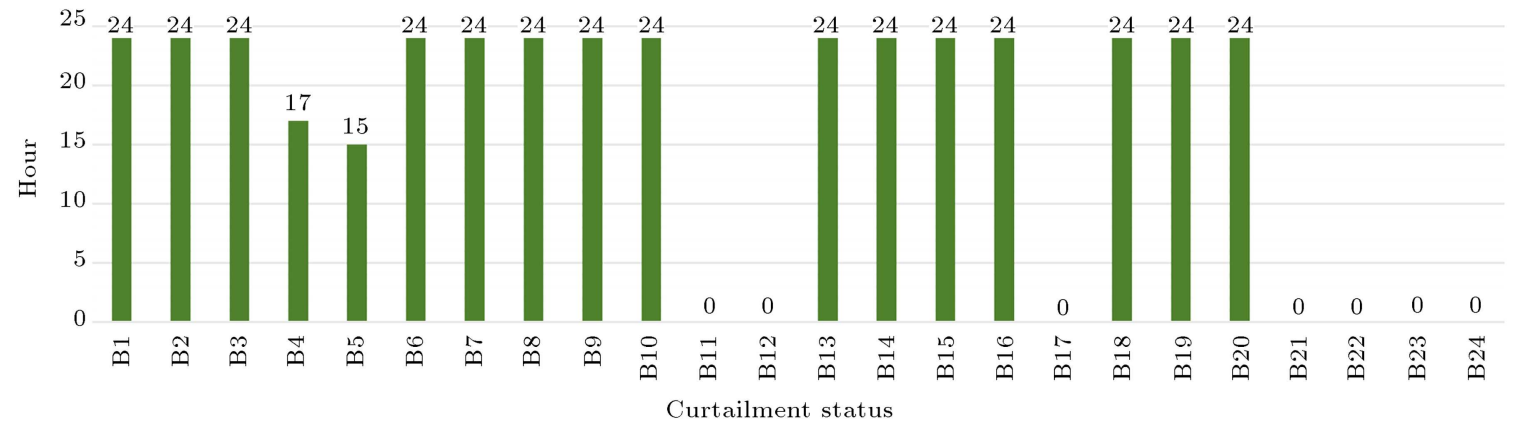

Figure 11. The load curtailment status for the 24-bus system.

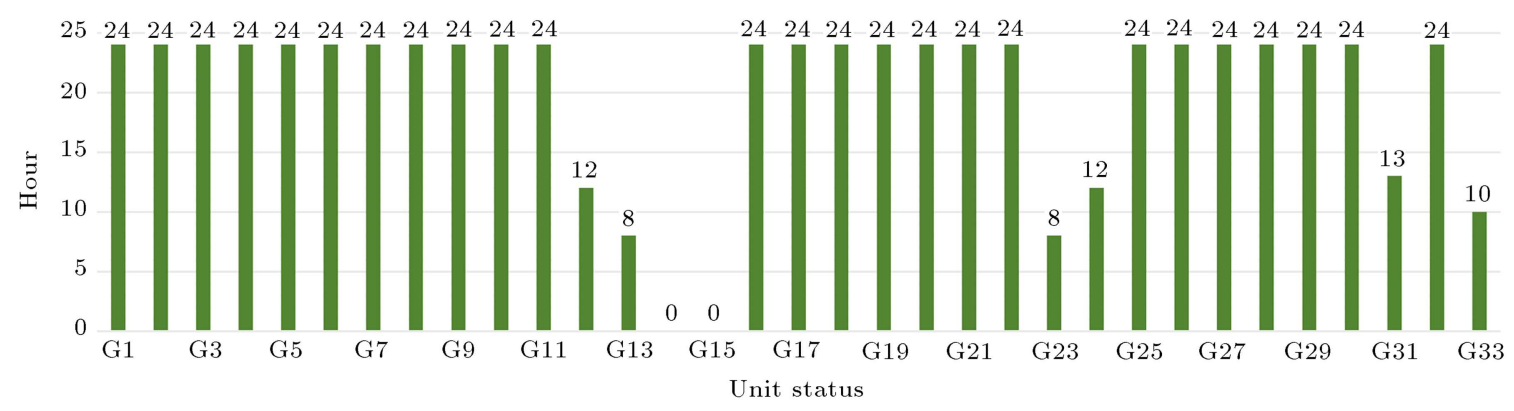

Figure 12. The unit status for the 24-bus system with the DR. 
Table 4. The DR effects on the demand reduction for the 24-bus system.

\begin{tabular}{lcccccccc}
\hline & \multicolumn{7}{c}{ Hour } \\
\cline { 2 - 9 } D1 (MW) & $\mathbf{T 1}$ & $\mathbf{T 2}$ & $\mathbf{T 3}$ & $\mathbf{T 4}$ & $\mathbf{T 5}$ & $\mathbf{T 6}$ & $\mathbf{T 7}$ & T8 \\
\cline { 2 - 9 } D2 (MW) & 1775.84 & 1669.815 & 1590.3 & 1563.795 & 1563.795 & 1590.3 & 1961.37 & 2279.43 \\
DR (MW) & 1673.25 & 1579.002 & 1508.013 & 1482.417 & 1482.417 & 1506.27 & 1795.215 & 2136.497 \\
\cline { 2 - 9 } & $\mathbf{T 9}$ & $\mathbf{T 1 0}$ & $\mathbf{T 1 1}$ & $\mathbf{T 1 2}$ & $\mathbf{T 1 3}$ & $\mathbf{T 1 4}$ & $\mathbf{T 1 5}$ & $\mathbf{T 1 6}$ \\
\cline { 2 - 9 } D1 (MW) & 2517.98 & 2544.48 & 2544.48 & 2517.975 & 2517.975 & 2517.975 & 2464.965 & 2491.47 \\
D2 (MW) & 166.797 & 169.385 & 169.385 & 166.797 & 166.797 & 166.797 & 161.82 & 165.147 \\
DR (MW) & 2351.18 & 2375.095 & 2375.095 & 2351.178 & 2351.178 & 2351.178 & 2303.145 & 2326.323 \\
\cline { 2 - 9 } & $\mathbf{T 1 7}$ & $\mathbf{T 1 8}$ & $\mathbf{T 1 9}$ & $\mathbf{T 2 0}$ & $\mathbf{T 2 1}$ & $\mathbf{T 2 2}$ & $\mathbf{T 2 3}$ & $\mathbf{T 2 4}$ \\
\cline { 2 - 9 } D1 (MW) & 2624 & 2650.5 & 2650.5 & 2544.48 & 2411.955 & 2199.915 & 1934.865 & 1669.815 \\
D2 (MW) & 177.339 & 180.049 & 180.049 & 169.448 & 156.131 & 134.991 & 113.456 & 91.954 \\
DR (MW) & 2446.66 & 2470.451 & 2470.451 & 2375.032 & 2255.824 & 2064.924 & 1821.409 & 1577.861 \\
\hline
\end{tabular}

Table 5. A complete comparison of all case studies.

\begin{tabular}{cccc}
\hline 6-bus system & Operational cost & Cost reduction & Time \\
\hline SCUC in base case & $137491.924 \$$ & $0 \$$ & $3 \mathrm{~min}$ \\
SCUC with DR & $124421.209 \$$ & $13070.715 \$$ & $5 \mathrm{~min}$ \\
\hline 24-bus system & Operational cost & Cost reduction & Time \\
\hline SCUC in base case & $373869.23 \$$ & $0 \$$ & $18 \mathrm{~min}$ \\
SCUC with DR & $349897.73 \$$ & $23971.5 \$$ & $24 \mathrm{~min}$ \\
\hline $\mathbf{1 1 8}$-bus system & Operational cost & Cost reduction & Time \\
\hline SCUC in base case & $1067031.191 \$$ & $0 \$$ & $67 \mathrm{~min}$ \\
SCUC with DR & $1049223.153 \$$ & $17808.038 \$$ & $79 \mathrm{~min}$ \\
\hline
\end{tabular}

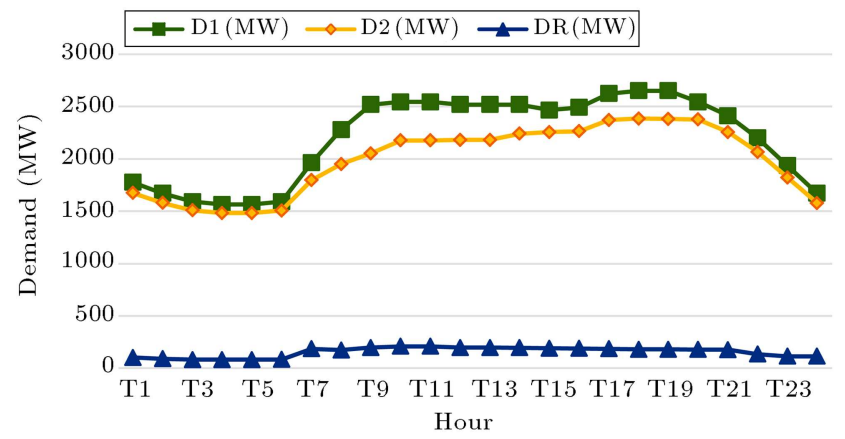

Figure 13. The DR effects on the demand curve for the 24-bus system.

According to the results, the DR incorporation is low in the first hours; hence, the demand curtailment is low. By achieving the peak load, the participation of DR increases; therefore, more demand curtailment occurs.

\subsection{A 118-bus system}

This IEEE test system has 54 generation units, 186 lines, and 9 tap-changers with the maximum peak load of $6000 \mathrm{MW}$ in a day. The total operation cost and the processing time after solving SCUC problem in the absence of the DR are obtained as $\$ 1,067,031.191$ and $67 \mathrm{~min}$, respectively. After incorporating the DR into the SCUC problem, the total operation cost and the processing time are obtained as $\$ 1,049,223.153$ and $79 \mathrm{~min}$, undergoing a reduction of $\$ 17808.038$ in comparison to that in the base case.

According to a complete comparison of all case studies shown in Table 5, the effectiveness of this approach in reducing the costs is proven.

\section{Conclusion}

In this paper, a powerful and generalized method, called benders' decomposition, was used to model the DR in SCUC problem. This mixed integer problem was applied to different cases containing a large case. The application of the DR to SCUC would effectively increase the social welfare. In summary, the results showed that the DR had the following merits in SCUC problem:

- Peak demand reduction; 
- Reduction in average system LMP;

- Increase in social welfare;

- Improved system reliability;

- Improvement of the environmental factors.

\section{Nomenclature}

$i$

$t$
Index for buses

Index for unit

Index for buses

Superscript for fixed loads

Superscript for responsive loads

Number of buses

Number of units

Number of periods (hour)

Ramp-down rate limit of unit $i$

Ramp-up rate limit of unit $i$

Production cost function for unit $i$

Commitment state of unit $i$ at time $t$

System losses at time $t$

Power production of unit $i$ at time $t$

Lower real power generation of unit $i$

Upper real power generation limit of unit $i$

System spinning reserve at time $t$

\section{System operating reserve at time $t$}

Spinning reserve of unit $i$ at time $t$

Startup cost of unit $i$ at time $t$

Shutdown cost of unit $i$ at time $t$

Minimum off time of unit $i$

Minimum on time of unit $i$

Off time of unit $i$ at time $t$

On time of unit $i$ at time $t$

Permissible real power adjustment of unit $i$

Maximum load curtailment at bus $b$

Fixed load at bus $b$ at time $t$

Responsive load at bus $b$ at time $t$

Submitted responsive load at bus $b$ at time $t$

Minimum load curtailment at bus $b$ at time $t$ Down-rate limit of load at bus $b$ Up-rate limit of load at bus $b$

Minimum on time of load at bus $b$ at time $t$

Minimum off time of load at bus $b$ at time $t$

$\begin{array}{ll}X_{b t}^{o n} & \text { On time of load at bus } b \text { at time } t \\ X_{b t}^{o f f} & \text { Off time of load at bus } b \text { at time } t \\ C B_{b t} & \text { Consumption benefit at bus } b \text { at time } t \\ D_{b t} & \text { Total load at bus } b \text { at time } t \\ V_{b t} & \text { Curtailment state of load at bus } b \text { at } \\ W_{t} & \text { time } t \\ \mu_{b t}, \pi_{b t} & \text { Power mismatch at time } t \\ \wedge & \text { Dual variable } \\ & \text { Given variable }\end{array}$

\section{References}

1. Shahidehpour, M., Yamin, H., and Li, Z. "Market operations in electric power systems: Forecasting, scheduling, and risk management", Market Overview in Electric Power Systems, pp. 1-20 (2002).

2. Conejo, A.J., Castillo, E., Minguez, R., and GarciaBertrand, R., Decomposition Techniques in Mathematical Programming: Engineering and Science Applications, Springer Science \& Business Media (2006).

3. Bakirtzis, A.G., Ziogos, N.P., Tellidou, A.C., and Bakirtzis, G.A. "Electricity producer offering strategies in day-ahead energy market with step-wise offers", IEEE Transactions on Power Systems, 22(4), pp. 1804-1818 (2007).

4. Nikzad, M. and Mozafari, B. "Reliability assessment of incentive-and priced-based demand response programs in restructured power systems", International Journal of Electrical Power \& Energy Systems, 56, pp. 83-96 (2014).

5. Zhang, D., Li, S., Sun, M., and O'Neill, Z. “An optimal and learning-based demand response and home energy management system", IEEE Transactions on Smart Grid, 7(4), pp. 1790-1801 (2016).

6. Nan, S., Zhou, M., and Li, G. "Optimal residential community demand response scheduling in smart grid", Applied Energy, 210, pp. 1280-1289 (2017).

7. Larsen, E.M., Pinson, P., Leimgruber, F., and Judex, F. "Demand response evaluation and forecasting methods and results from the ecogrid eu experiment", Sustainable Energy, Grids and Networks, 10, pp. 75-83 (2017).

8. Bui, V.-H., Hussain, A., and Kim, H.-M. "A multiagent-based hierarchical energy management strategy for multi-microgrids considering adjustable power and demand response", IEEE Transactions on Smart Grid, 9, pp. 1323-1333 (2018).

9. Dehghanpour, K., Nehrir, H., Sheppard, J., and Kelly, N. "Agent-based modeling of retail electrical energy markets with demand response", IEEE Transactions on Smart Grid, 9, pp. 3465-3475 (2018). 
10. Wang, F., Xu, H., Xu, T., Li, K., Shafie-Khah, M., and Catalão, J.P. "The values of market-based demand response on improving power system reliability under extreme circumstances", Applied Energy, 193, pp. 220231 (2017).

11. David, A. and Li, Y. "Effect of inter-temporal factors on the real time pricing of electricity", IEEE Transactions on Power Systems, 8(1), pp. 44-52 (1993).

12. Samimi, A., Nikzad, M., and Siano, P. "Scenariobased stochastic framework for coupled active and reactive power market in smart distribution systems with demand response programs", Renewable Energy, 109, pp. 22-40 (2017).

13. Goel, L., Wu, Q., and Wang, P. "Nodal price volatility reduction and reliability enhancement of restructured power systems considering demand-price elasticity", Electric Power Systems Research, 78(10), pp. 16551663 (2008).

14. Zhang, D., Wang, Y., and Luh, P.B. "Optimization based bidding strategies in the deregulated market", in, Power Industry Computer Applications, 1999. PICA'99, Proceedings of the 21st 1999 IEEE International Conference IEEE (1999).

15. Arroyo, J.M. and Conejo, A.J. "Multiperiod auction for a pool-based electricity market", IEEE Transactions on Power Systems, 17(4), pp. 1225-1231 (2002).

16. Contreras, J., Candiles, O., De La Fuente, J.I., and Gomez, T. "Auction design in day-ahead electricity markets", IEEE Transactions on Power Systems, 16(3), pp. 409-417 (2001).

17. Su, C. and Kirschen, D. "Direct participation of demand-side in a pool-based electricity market", POWER SYSTEM TECHNOLOGY-BEIJING-, 31(20), p. 7 (2007).

18. Kirschen, D.S., Strbac, G., Cumperayot, P., and de Paiva Mendes, D. "Factoring the elasticity of demand in electricity prices", IEEE Transactions on Power Systems, 15(2), pp. 612-617 (2000).

19. Nguyen, D.T., Negnevitsky, M., and De Groot, M. "Pool-based demand response exchange-concept and modeling", IEEE Transactions on Power Systems, 26(3), pp. 1677-1685 (2011).
20. Khodaei, A., Shahidehpour, M., and Bahramirad, S. "Scuc with hourly demand response considering intertemporal load characteristics", IEEE Transactions on Smart Grid, 2(3), pp. 564-571 (2011).

21. Fu, Y., Shahidehpour, M., and Li, Z. "Securityconstrained unit commitment with Ac constraints", IEEE Transactions on Power Systems, 20(3), pp. 1538-1550 (2005).

22. Wu, H., Guan, X., Zhai, Q., and Ye, H. "A systematic method for constructing feasible solution to scuc problem with analytical feasibility conditions", IEEE Transactions on Power Systems, 27(1), pp. 526-534 (2012).

23. Bussieck, M.R. and Vigerske, S. "MINLP solver software", Wiley encyclopedia of operations research and management science (2010).

\section{Biographies}

Ebrahim Zarei received his MSc degree from the Electrical Engineering Department of Shahid Bahonar University of Kerman/Iran. His research interests include operation, scheduling power plants, smart grids, renewable energy modeling, analysis and control.

Mohammad Hasan Hemmatpour received his BS, $\mathrm{MSc}$, and $\mathrm{PhD}$ degrees in Electrical Engineering from the Shahid Bahonar University of Kerman, Iran as an honor student in 2009, 2012, and 2016, respectively. Currently, he is an Assistant Professor at the Electrical Engineering Department of Jahrom University/Iran. His research interests include operation, controls and analysis of microgrids, optimization techniques, and renewable energy modeling.

Mohsen Mohammadian received her BS degree in Electrical Engineering from Sharif University, and his MSc and PhD degrees in Electrical Engineering from K.N. Toosi University, Tehran. He is as an Assistant Professor from the Electrical Engineering Department of Shahid Bahonar University of Kerman, Iran. His current research interests include non-linear control, intelligent control, and control of complex systems, such as hybrid electric vehicles and power systems. 